\title{
Effects of PI3KY overexpression in the hippocampus on synaptic plasticity and spatial learning
}

\author{
Jun-Hyeok Choi ${ }^{1 \dagger}$, Pojeong Park ${ }^{2+}$, Gi-Chul Baek ${ }^{1 \dagger}$, Su-Eon Sim², SukJae Joshua Kang ${ }^{2}$, Yeseul Lee ${ }^{2}$, Seo-Hee Ahn², \\ Chae-Seok Lim', Yong-Seok Lee ${ }^{3}$, Graham L Collingridge ${ }^{4}$ and Bong-Kiun Kaang ${ }^{1,2^{*}}$
}

\begin{abstract}
Previous studies have shown that a family of phosphoinositide 3-kinases (PI3Ks) plays pivotal roles in the brain; in particular, we previously reported that knockout of the $\gamma$ isoform of PI3K (PI3KY) in mice impaired synaptic plasticity and reduced behavioral flexibility. To further examine the role of PI3KY in synaptic plasticity and hippocampusdependent behavioral tasks we overexpressed p110y, the catalytic subunit of PI3KY, in the hippocampal CA1 region. We found that the overexpression of p110y impairs NMDA receptor-dependent long-term depression (LTD) and hippocampus-dependent spatial learning in the Morris water maze (MWM) task. In contrast, long-term potentiation (LTP) and contextual fear memory were not affected by p110y overexpression. These results, together with the previous knockout study, suggest that a critical level of PI3KY in the hippocampus is required for successful induction of LTD and normal learning.
\end{abstract}

\section{Introduction}

Phosphoinositide 3-kinases (PI3Ks) are involved in various biological processes such as growth, proliferation and differentiation of cells $[1,2]$. PI3Ks produce various phosphorylated phosphoinositides, including PtdIns3P, PtdIns (3,4)P2, PtdIns(3,5)P2, and PtdIns(3,4,5)P3; all of which function in membrane trafficking and signal transduction $[3,4]$. The PI3K family is grouped into three classes, I, II and III, according to their structures and substrates. The class I PI3Ks are further divided into IA and IB classes based on the similarities of their sequences. PI3K $\gamma$, the sole member of class IB, is a heterodimer of the p110y catalytic subunit and either p101 or p84 regulatory subunits, which are encoded by separate genes [1]. Unlike other types of PI3Ks, which have a widespread tissue distribution, $\mathrm{PI} 3 \mathrm{~K} \gamma$ is exclusively expressed in the immune system, the cardiovascular system, and the central nervous

\footnotetext{
* Correspondence: kaang@snu.ac.kr

${ }^{\dagger}$ Equal contributors

'Department of Biological Sciences, College of Natural Sciences, Seoul National University, Bldg 504 Rm 202, 599 Gwanangno, Gwanak-gu, Seoul 151-747, Korea

${ }^{2}$ Department of Brain and Cognitive Sciences, College of Natural Sciences, Seoul National University, Seoul, Korea

Full list of author information is available at the end of the article
}

system [2]. The roles of PI3K $\gamma$ have primarily been studied in the field of immunology where it was first identified in the study of polyoma viruses [3].

Although mounting evidence has revealed that the members of PI3K family are required for learning and memory [4-8], the isoform-specific roles of PI3Ks are still largely undetermined. Recently, we found that PI3K $\gamma$ knockout mice $\left(\right.$ pi3 $\left.\mathrm{kgg}^{-/-}\right)$had a selective impairment in NMDA receptor (NMDAR)-dependent LTD and reversal learning in the Morris water maze (MWM) task [9]. To investigate the function of PI3K $\gamma$ in more detail, we have now used a gain-of-function approach by overexpressing PI3K $\gamma$ and have studied hippocampus-dependent learning and synaptic plasticity. We found that NMDAR-LTD, but not NMDAR-LTP, was impaired in slices prepared from mice in which PI3KY had been overexpressed in the hippocampus. Moreover, performance in a spatial learning task was reduced by $\mathrm{PI} 3 \mathrm{~K} \gamma$ overexpression, while contextual fear memory was not affected. Our findings further support a role of PI3K $\gamma$ in some, but not all, forms of hippocampusdependent synaptic plasticity and spatial learning. 


\section{Materials and methods}

\section{AAV production and stereotactic viral injection}

All adeno-associated viruses (AAVs) were produced with AAV2/1 serotyped vectors and packaged using HEK293T cells. Viral titers were determined by quantitative realtime PCR (Prism 7300, Applied Biosystems, USA) with intercalater SYBR green (TAKARA, Japan).

All surgical procedures were conducted in sterile conditions and approved by the Institutional Animal Care and Use Committees of Seoul National University. Wild-type C57BL/6 male mice ( 8 weeks of age) were anesthetized by an intraperitoneal injection of ketamine and arranged in a stereotactic frame (Stoelting Co. USA). The hippocampal CA1 region (AP: $-1.8 \mathrm{~mm}, \mathrm{ML}: \pm 1.5 \mathrm{~mm}, \mathrm{DV}:-1.7 \mathrm{~mm}$ ) was targeted, and equivalent amounts of AAVs $\left(3.5 \times 10^{9}\right.$ particles in $1 \mu$ for flag-p110 $\gamma$ and tdTomato) were delivered bilaterally using a 10- $\mu$ l syringe pump (30 gauge, Hamilton Co., USA) at a rate of $6.0 \mu \mathrm{l} / \mathrm{h}$. After an additional $10 \mathrm{~min}$ of diffusion time, the needle was withdrawn and the scalp was sutured with black silk. AAV vectors were then expressed for 2 weeks.

Histology, immunohistochemistry and fluorescence imaging The mice were kept under isoflurane anesthesia and transcardially perfused with pre-chilled $4 \%$ paraformaldehyde (PFA) in PBS. Brains were fixed again in 4\% PFA at $4^{\circ} \mathrm{C}$ overnight and then dehydrated in $30 \%$ sucrosePBS solution at $4^{\circ} \mathrm{C}$ for 2 days. Coronal brain slices were prepared (30 $\mu \mathrm{m}$ for flag-p110, $50 \mu \mathrm{m}$ for tdTomato) by cryosectioning (Leica Ltd., Germany), 50- $\mu \mathrm{m}$ sections for tdTomato were mounted on a slide glass with Vectashield containing DAPI (Vector Lab, USA), and 30- $\mu$ m sections for flag-p110 $\gamma$ were stored in $50 \%$ glycerol in PBS at $-20^{\circ} \mathrm{C}$.

Flag-p110y was detected with anti-Flag antibody (Sigma, USA, Cat\# F7425, 1:1,000) and goat anti-rabbit Alexa Fluor 488 IgG (Invitrogen, USA, Cat\# A11008, 1:500). Fluorescent mages were acquired using an IX51 inverted fluorescent microscope (Olympus, Japan).

\section{Electrophysiology \\ Hippocampal slice preparation}

Transverse hippocampal slices $(400 \mu \mathrm{m})$ were prepared from C57BL/6 mice aged 12 weeks (24 days after AAV infusion). The animals were anaesthetized with isoflurane and decapitated for the removal of the brain. Brains were then placed in ice-chilled artificial cerebrospinal fluid (ACSF, $124 \mathrm{mM} \mathrm{NaCl}, 3 \mathrm{mM} \mathrm{KCl}, 26 \mathrm{mM} \mathrm{NaHCO}$, $1.25 \mathrm{mM} \mathrm{NaH} \mathrm{PO}_{4}, 10 \mathrm{mM} \mathrm{MgSO}, 10 \mathrm{mM}$ Glucose, $1 \mathrm{mM} \mathrm{CaCl}_{2}$, aerated with $95 \% \mathrm{O}_{2}$ and $\left.5 \% \mathrm{CO}_{2}\right)$. The hippocampus was removed from the brain while maintained in the cold ice solution and sliced using a VT1000 slicer (Leica Ltd., Germany). Hippocampal slices were transferred to ACSF containing $2 \mathrm{mM} \mathrm{MgSO}_{4}$ and $2 \mathrm{mM}$ $\mathrm{CaCl}_{2}$, where they were allowed to recover at $32^{\circ} \mathrm{C}$ for
$30 \mathrm{~min}$ and then maintained at $28^{\circ} \mathrm{C}$ before recordings were conducted.

\section{Slice electrophysiology}

Extracellular recordings were performed in an interface chamber (Campden Instrument Ltd., UK) maintained at $32^{\circ} \mathrm{C}$. ACSF (124 mM NaCl, $3 \mathrm{mM} \mathrm{KCl,} 26 \mathrm{mM}$ $\mathrm{NaHCO}_{3}, 1.25 \mathrm{mM} \mathrm{NaH} \mathrm{PO}_{4}, 2 \mathrm{mM} \mathrm{MgSO}, 10 \mathrm{mM}$ glucose, $2 \mathrm{mM} \mathrm{CaCl}$, aerated as above) was perfused continuously at a rate of $2 \mathrm{ml} / \mathrm{min}$. Standard extracellular recordings were performed in the CA1 region of the hippocampal slices to measure the slope of evoked field excitatory postsynaptic potentials (fEPSPs). Responses were obtained using an Axopatch 200B amplifier (Molecular Devices, US) and digitized with a Digidata 1322A A/D board at a sampling rate of $10 \mathrm{kHz}$. Recordings were monitored and analyzed using WinLTP (WinLTP Ltd. (winltp.com), and The University of Bristol, UK).

\section{Synaptic plasticity}

A bipolar stimulating electrode was placed in the stratum radiatum of the CA1 region. Baseline responses at a frequency of $0.033 \mathrm{~Hz}$ were obtained using a stimulus intensity (between 5 to $20 \mu \mathrm{A}$; $0.1 \mathrm{~ms}$ pulse width) that evoked responses of approximately $40 \%$ of the maximum fEPSP value. Following a stable baseline period of at least $20 \mathrm{~min}$, LTP and LTD were investigated by delivering high-frequency stimulation $(100 \mathrm{~Hz}, 1 \mathrm{~s})$ and lowfrequency stimulation $(1 \mathrm{~Hz}, 15 \mathrm{~min})$, respectively. In terms of depotentiation experiments, LTP was induced using three trains of theta burst stimulations (consisting of five pulses at $100 \mathrm{~Hz}$ and repeated five times at $5 \mathrm{~Hz}$ ), with a $10 \mathrm{~s}$ inter-train interval. After $30 \mathrm{~min}$ of LTP induction, low-frequency stimulation ( $2 \mathrm{~Hz}, 10 \mathrm{~min}$ ) was given to depotentiate the responses.

\section{Statistical analysis}

All experimental procedures were performed in a blind fashion. The data are presented as the mean \pm SEM, and statistical significance was determined using Student's t-test, and the level of significance was set at ${ }^{*} \mathrm{p}<0.05$ and $*$ p $<0.01$. To compare the level of synaptic plasticity between the groups, the average of fEPSP responses obtained from the last 5 min period was used.

\section{Western immunoblotting}

For western immunoblotting, we injected flag-p110 $\gamma$ or tdTomato expressing AAV, together with GFP expressing $\mathrm{AAV}$ in the CA1 region of 3 weeks-old C57BL/6 male mice. 2 weeks after injection, CA1 regions expressing green fluorescence were dissected and recovered in ACSF maintained at $32^{\circ} \mathrm{C}$ for $2 \mathrm{~h}$. CA1 mini slices were treated with NMDA $(20 \mu \mathrm{M})$ for 3 min to induce chemical LTP and immediately snap-frozen in liquid nitrogen. Slices 
were homogenized using a dounce homogenizer with lysis buffer (10 mM Tris- $\mathrm{HCl}, 150 \mathrm{mM} \mathrm{NaCl}, 1 \mathrm{mM}$ EDTA, $1 \%$ NP-40, 0.1\% SDS, pH 7.5) containing both protease inhibitor cocktail (Roche) and phosphatase inhibitor cocktail (Roche). Samples were analyzed by western blotting using the following antibodies: anti-phospho-p38 MAPK (Cell Signaling) and anit-p38 MAPK (Cell Signaling).

\section{Behavioral experiment}

Male C57BL/6 mice were housed two or four per cage and maintained on a 12/12 h light-dark cycle. Food and water were provided ad libitum.

\section{Open field test}

The mice were exposed to the open field box, a square opaque white box $(40 \times 40 \times 40 \mathrm{~cm})$, for 10 min under dim light. Locomotor activity was monitored and analyzed with EthoVision 3.1 (Noldus, Netherlands).

\section{Elevated plus maze task}

The elevated plus maze was made of white Plexiglas and consisted of two opposing open arms and two closed arms. All arms were $150 \times 5 \mathrm{~cm}$ (length $\times$ width) and closed arms were enclosed with $20-\mathrm{cm}$ walls. The maze was placed $30 \mathrm{~cm}$ above the floor. Mice were placed in the center of the maze and tracked for $5 \mathrm{~min}$ by EthoVision 3.1 (Noldus, Netherlands).

\section{Morris Water Maze (MWM) task}

The water maze was a circular opaque grey tank, $140 \mathrm{~cm}$ in diameter, $100 \mathrm{~cm}$ in height, filled with water containing small amount of white paint to hide the platform $\left(22 \sim 23^{\circ} \mathrm{C}\right)$ to a depth of $30 \mathrm{~cm}$. The maze was surrounded by four distinct visual cues, and all procedures were performed under dim light. A circular, $10 \mathrm{~cm}$ in diameter, Perspex platform was positioned in the middle of the virtual quadrant (distance of $35 \mathrm{~cm}$ from the edge and $35 \mathrm{~cm}$ to the center of the maze) and submerged approximately $1.5 \mathrm{~cm}$ below the surface of the water. Each training session consisted of four 60-s trials with four different starting points that were randomly selected and equally distributed among the four quadrants. The mice underwent one training session per day. When the mice reached the platform, they were allowed to remain on the platform for $20 \mathrm{~s}$ and then transferred to a holding-cage for $60 \mathrm{~s}$. If a mouse failed to locate the platform within $60 \mathrm{~s}$, it was manually guided to the platform. For the probe test, on day 6 , the mice were allowed $60 \mathrm{~s}$ of free-swimming without the platform. All experiments were recorded and tracked using Ethovision 3.1 (Noldus, Netherlands).

\section{Contextual fear conditioning}

Contextual fear memory was measured in a standard chamber (Freeze Frame, Coulbourn) after 4 days of handling.
The mice were placed in the fear chamber for $5 \mathrm{~min}$. After $2 \mathrm{~min}$, the mice were given three consecutive foot shocks ( $2 \mathrm{~s}, 0.24 \mathrm{~mA}, 1 \mathrm{~min}$ intervals). After $24 \mathrm{~h}$, the mice were again placed in the same chamber for memory retrieval.

\section{Results}

\section{Electrophysiological properties of $\mathrm{p} 110 \mathrm{\gamma}$ overexpressed} hippocampal slices

p110 $\gamma$ or control red fluorescent protein, tdTomato, was delivered to the hippocampal CA1 region by AAV vector, driven by a CaMKII $\alpha$ promoter for neuron-specific expression. Flag-tagged $\mathrm{p} 110 \mathrm{\gamma}$ as well as the tdTomato were expressed in both the soma and the dendrites of the CA1 neurons (Figure 1A).

We first investigated the effects of $\mathrm{p} 110 \gamma$ overexpression on electrophysiological properties in the CA1 region of hippocampus. Analysis of basal synaptic transmission showed no significant difference in input/output relationships in PI3K $\gamma$ overexpressed slices compared to the control group (PI3K $\gamma$ overexpressed: $n=9,6$ animals; control: $\mathrm{n}=11,7$ animals, Figure 1B, C). Paired-pulse facilitation (PPF) was also unaffected, indicating normal presynaptic release probability (PI3K $\gamma$ overexpressed: $n=9,6$ animals; control: $\mathrm{n}=11,7$ animals, Figure 1D). Taken together, these results indicate that basal synaptic transmission was not changed by $110 \gamma$ overexpression in the hippocampal Schaffer collateral (SC)-CA1 synapses.

We then probed the role of p110 $\gamma$ overexpression with regards to activity-dependent synaptic plasticity. The amount of LTP induced by a brief tetanus $(100 \mathrm{~Hz}, 1 \mathrm{~s})$ was not significantly different between the p110 $\gamma$ overexpression group and the control group (PI3K $\gamma$ overexpressed: $n=11,127 \pm 6 \%$ of baseline, 6 animals; control: $n=12,123 \pm 4 \%$ of baseline, 6 animals, Figure 1E). On the other hand, LTD was barely induced by repetitive low-frequency stimulations (LFS, $1 \mathrm{~Hz}, 15 \mathrm{~min}$ ) in the $\mathrm{p} 110 \mathrm{\gamma}$ overexpressed slices compared to the tdTomato expressed control group (Figure 1F). Field EPSP slope measured $1 \mathrm{hr}$ after LTD induction for the $\mathrm{p} 110 \mathrm{\gamma}$ and control group was $96 \pm 5 \%(\mathrm{n}=10,7$ animals $)$ and $77 \pm$ $4 \%$ of baseline ( $n=12,8$ animals), respectively (Student's $\mathrm{t}$-test, $\mathrm{p}<0.01)$. Moreover, we identified that the reversal of LTP (i.e., depotentiation) by a train of LFS $(2 \mathrm{~Hz}$, $10 \mathrm{~min}$ ) was reduced in the $\mathrm{p} 110 \mathrm{\gamma}$ overexpression group ( $\mathrm{n}=10,7$ animals, $127 \pm 6$ of pre-LTP baseline) in contrast to the control group ( $\mathrm{n}=12,8$ animals, $85 \pm 11 \%$ of baseline), indicating synapses become less plastic following $\mathrm{p} 110 \gamma$ overexpression (Student's t-test, $\mathrm{p}<0.01$, Figure $1 \mathrm{G}$ ).

\section{Molecular mechanisms of impaired NMDAR-LTD in p110 $\gamma$-overexpressed hippocampal slices}

We next examined the effect of $\mathrm{p} 110 \mathrm{\gamma}$ overexpression on the downstream pathway involved in NMDAR-LTD. p38 MAPK is known to be involved in NMDAR-LTD 


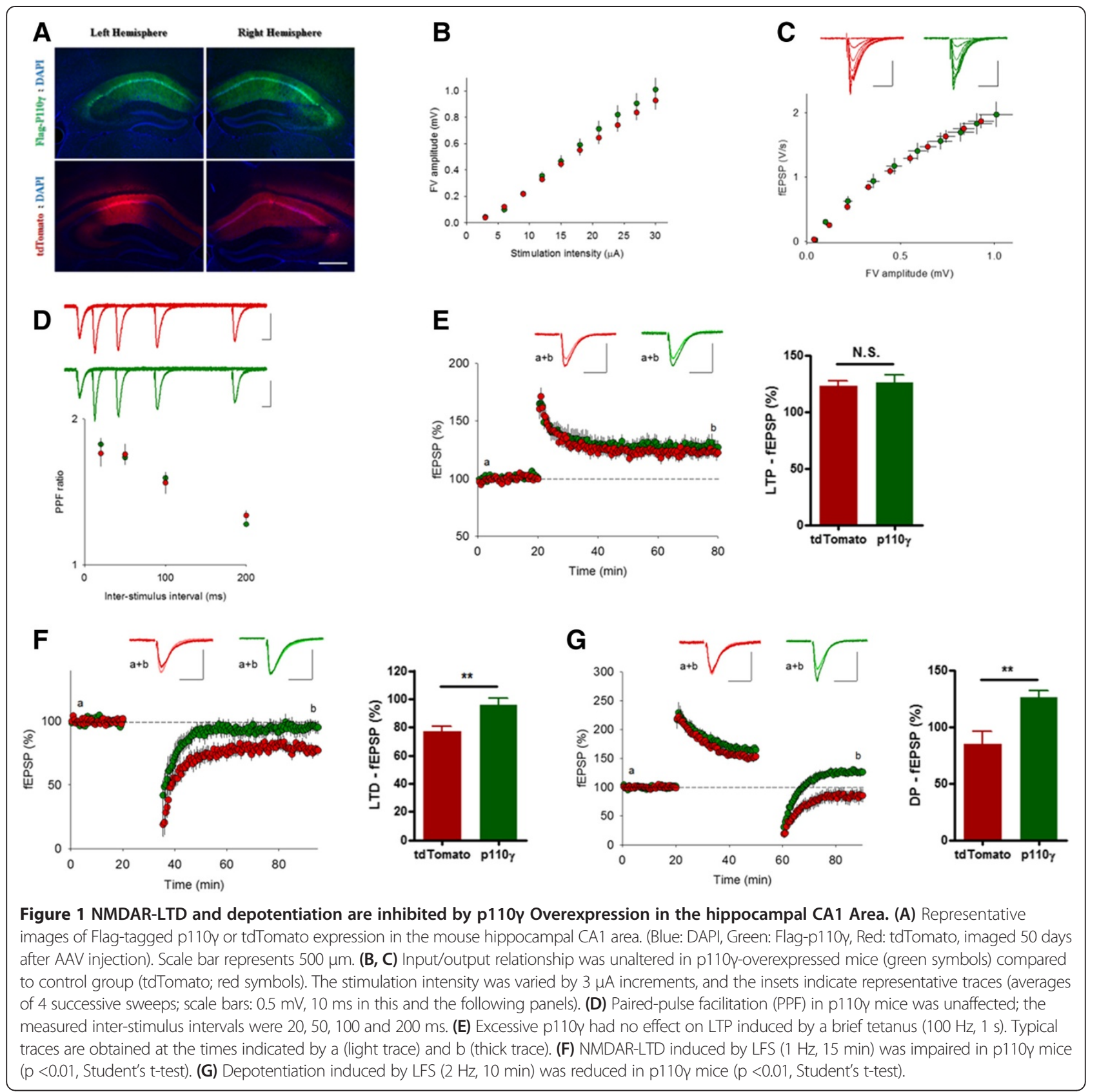

through Rap1 signaling pathway and active Rap1 directly binds to p110y [10-12]. In our previous study, we found that $\mathrm{p} 110 \mathrm{y}$ deletion reduced the phosphorylation of $\mathrm{p} 38$ MAPK induced by chemical LTD [9]. We performed similar chemical LTD experiments with tdTomato- or p110yoverexpressing hippocampal slices. We first confirmed that p110y overexpression itself did not affect the basal phosphorylation level of p38 MAPK $(n=5$, Figure $2 A, B)$. The phosphorylation of p38 MAPK (p-p38) was significantly increased in response to the chemical LTD induction in the tdTomato-expressing group (Figure 2A, C). However, the increase in p38 MAPK phosphorylation associated with chemical LTD was impaired in p110yoverexpressing group (Figure 2A, D). These results support the finding that NMDAR-LTD is impaired by p110y overexpression and suggest that this might be due to impairment in p38 MAPK signaling.

\section{Effect of $\mathrm{p} 110 \mathrm{\gamma}$ overexpression in the hippocampal CA1 region on anxiety and locomotor activity}

Next, we examined the effect of p110y overexpression on basal behavioral tasks. We performed a series of behavioral experiments where basal anxiety and locomotion were measured. In the elevated plus maze (EPM) 
A

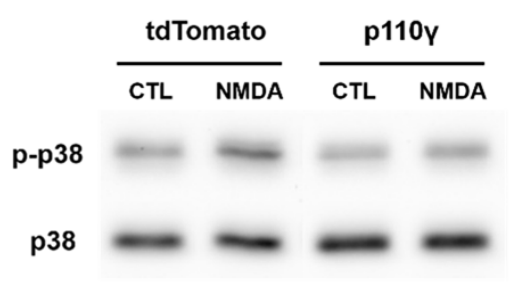

C

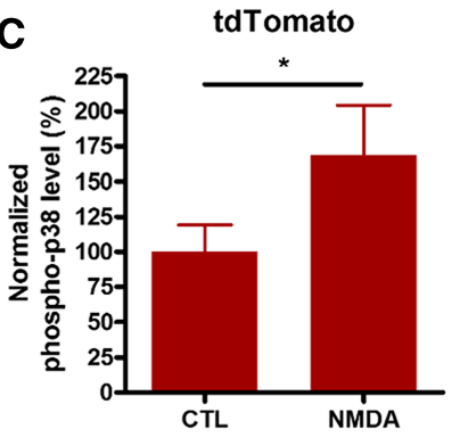

B
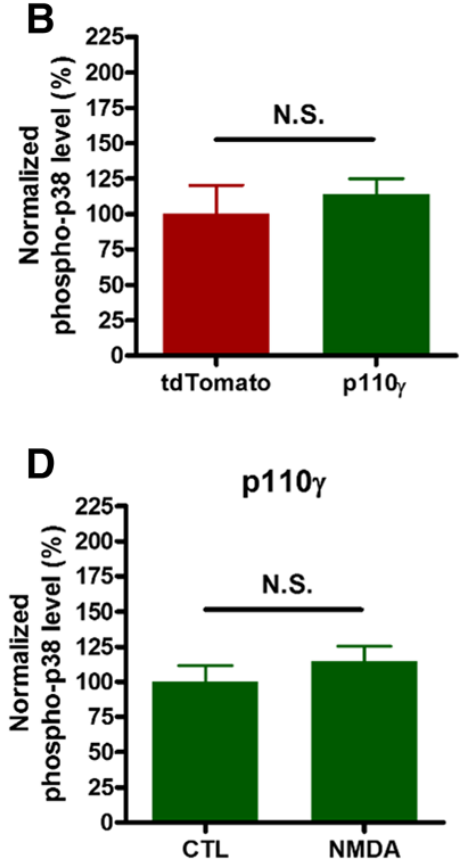

Figure 2 Impaired induction of p38 MAPK phosphorylation by chemical LTD in p110y-overexpressed hippocampal CA1 region. (A) Representative phospho/total p38 MAPK western blot from tdTomato- or p110y-overexpressed slices after chemical LTD induction (NMDA treatment). (B) Normalized basal phosphorylation level of p38 MAPK from tdTomato- or p110y-overexpressed slices. (C) Normalized phosphorylation level of p38 MAPK from tdTomato-overexpressed slices after chemical LTD induction ( $n=5, p<0.05$, paired Student's t-test). (D) Normalized phosphorylation level of p38 MAPK from p110y-overexpressed slices, after chemical LTD induction ( $n=5$, NS, paired Student's t-test).

test, which is a well-established method to assess anxiety level in rodents, the p110y-overexpressed group exhibited a similar level of anxiety compared to the control group (there was a tendency to be more anxious in the p110y-overexpressed group without any statistical significance; Figure 3A). In addition, the anxiety level and the locomotive activity of the p110y-overexpressed group were similar to those of the control group in the open field test (OFT) (Figure 3B, C), indicating that the exogenous expression of $\mathrm{p} 110 \gamma$ does not affect basal rodent behaviors such as anxiety and locomotion.

\section{Effect of $\mathrm{p} 110 \gamma$ overexpression on hippocampus-dependent learning tasks}

We next investigated the effect of $\mathrm{p} 110 \gamma$ overexpression on hippocampus-dependent learning tasks. Our previous study showed that without pre-exposure to the context in contextual fear conditioning, the freezing level was reduced in $p i 3 \mathrm{kcg}^{-/-}$mice [9]. Furthermore, in the reversal learning session of the Morris Water Maze (MWM) task, time spent in the old-target quadrant where the platform was located during the initial training sessions was increased in $\mathrm{pi} 3 \mathrm{kgg}^{-/-}$mice [9]. These results suggested that $\mathrm{PI} 3 \mathrm{~K} \gamma$ plays a critical role in hippocampus-dependent learning tasks. Therefore, we wanted to determine whether contextual fear memory and spatial learning were affected by $\mathrm{p} 110 \mathrm{\gamma}$ overexpression. We performed contextual fear conditioning and MWM test with the same protocols used in the previous study [9]. In the contextual fear memory test, the p110 $\gamma$-overexpressed group showed equivalent freezing levels compared to the control group (Figure 4A), suggesting that $\mathrm{p} 110 \mathrm{\gamma}$ overexpression in the hippocampus did not enhance or impair fear memory formation. However, spatial learning in the MWM test was significantly disrupted by overexpression of p110y (Figure 4B). In addition, the $\mathrm{p} 110 \mathrm{\gamma}$ overexpression group exhibited a tendency to spend less time in the target quadrant and fewer number of platform crossings during the probe test on day 6, suggesting that $\mathrm{p} 110 \mathrm{\gamma}$ overexpression has a negative impact on spatial memory (Figure 4C, D).

\section{Discussion}

We investigated the behavioral and electrophysiological effects of PI3K $\gamma$ overexpression in the hippocampal CA1 region of mice. Our previous experiments with $\mathrm{Pik} 3 \mathrm{cg}^{-/-}$ mice demonstrated that PI3K $\gamma$ was critically involved in NMDA receptor-dependent LTD (NMDAR-LTD) and reversal learning in the MWM [9]. To further elucidate the role of PI3K $\gamma$ in synaptic plasticity and learning, we used an AAV-mediated genetic delivery of p110 $\gamma$, a catalytic subunit of PI3K $\gamma$, to the hippocampal CA1 neurons.

We found that the overexpression of $\mathrm{p} 110 \gamma$ in the CA1 region disrupted NMDAR-LTD and depotentiation of potentiated synapses and that this effect was associated with 

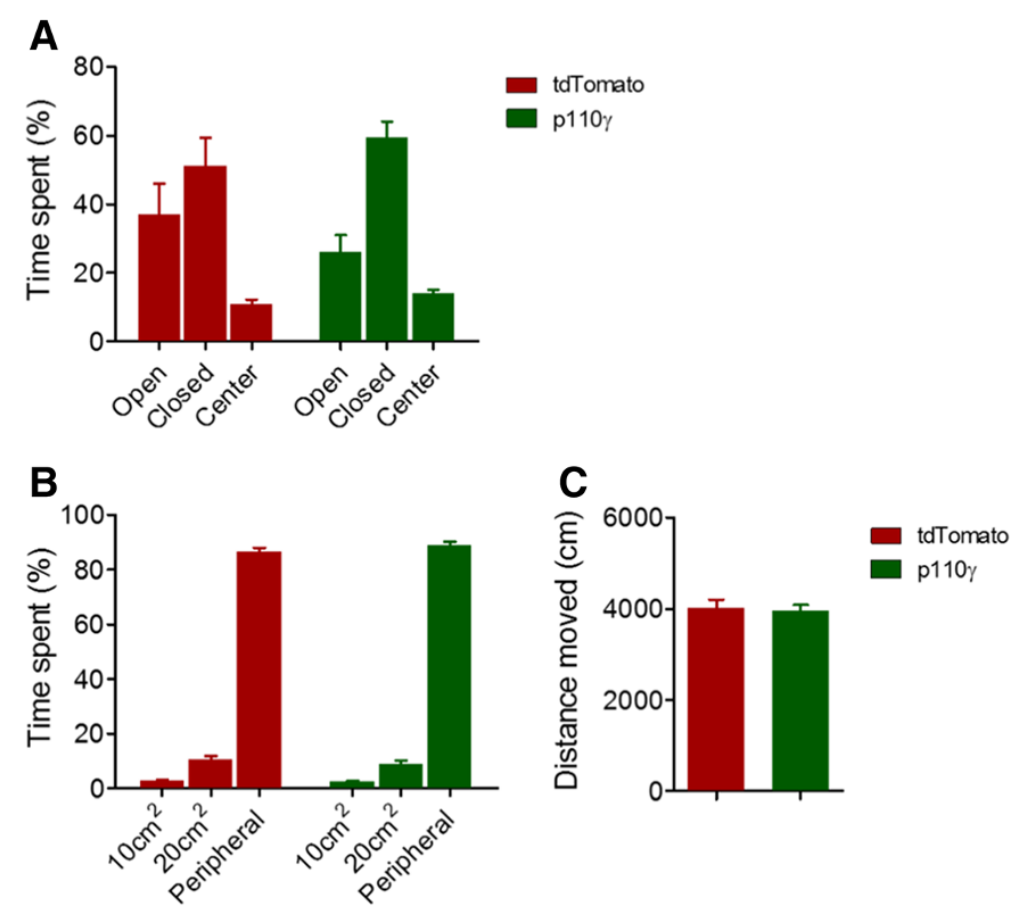

Figure 3 Effect of p110y overexpression on basal anxiety and locomotion. (A) Basal anxiety levels of p110y-or tdTomato-overexpressed mice in the elevated plus maze (EPM) test (Flag-p110y, $n=12$; tdTomato, $n=9$, tested at 2 weeks after AAV injection). (B) Basal locomotive activity in the open field (OFT) test (Flag-p110y, $\mathrm{n}=14$; tdTomato, $\mathrm{n}=12$, tested at 15 days after AAV injection). (C) Total distance moved ( $\mathrm{cm}$ ) during $100 \mathrm{~s}$ in the OFT.
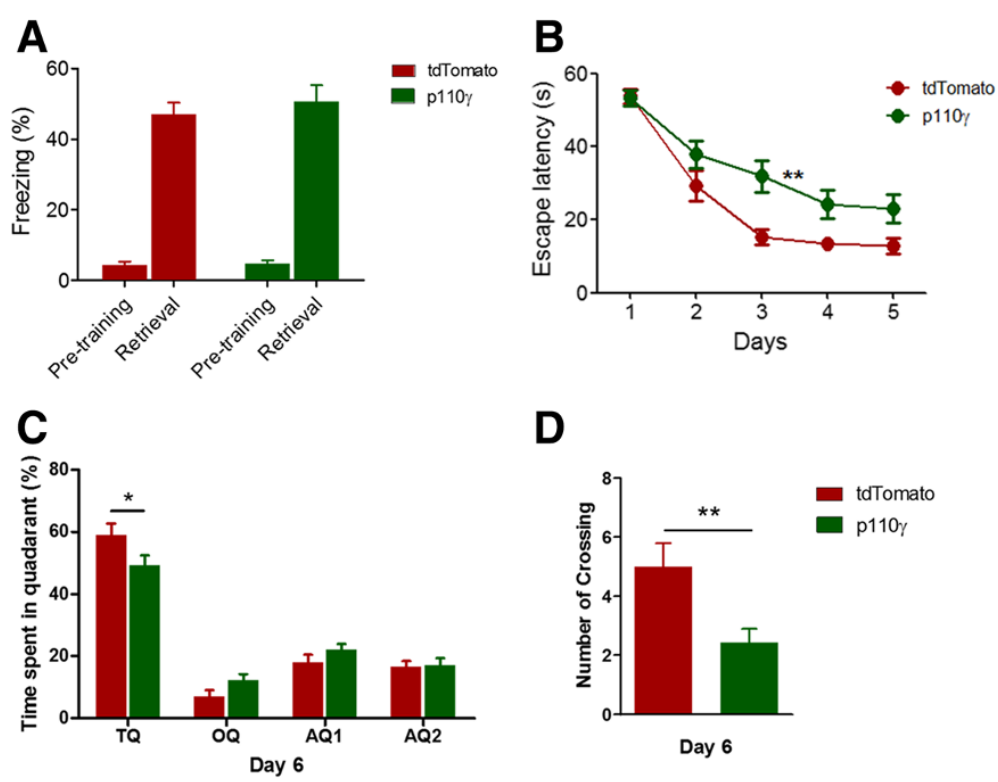

Figure 4 Spatial learning in the Morris Water Maze (MWM) Task was reduced in p110 $\boldsymbol{\gamma}^{-} \boldsymbol{\gamma}-\boldsymbol{\gamma}-$. (A) Contextual fear memory of p110 $\gamma$ - or tdTomato-expressed mice (Flag-p110y, $n=14$; tdTomato, $n=12$ ). (B) Average escape time traveled to the hidden platform (mean \pm sem) in the MWM test (flag-p110 $\gamma, n=14$; tdTomato, $n=12, F=8.25,{ }^{* *} p<0.01$, repeated measures two-way ANOVA). (C) The average percentage of time spent in the target quadrant ( $T Q,{ }^{*} p<0.05$, repeated measures two-way ANOVA), the opposite quadrant $(\mathrm{OQ})$ and two adjacent quadrants (AQ) during the probe trial given on day 6 of the MWM task. (D) Total number of crossings of the location of the platform $\left({ }^{* *} p<0.01\right.$, Student's t-test) during the probe trials. 
a reduced phosphorylation level of p38 MAPK in response to LTD induction. In contrast, LTP in the p110y overexpression group was normal. Hippocampus-dependent spatial memory in the Morris water maze (MWM) task was also impaired. The finding that a similar impairment in NMDAR-LTD results from either p110y knockout or overexpression indicates that an appropriate level of $\mathrm{p} 110 \mathrm{y}$ is required for normal LTD. This idea is further supported by the similar effects on p38 MAPK phosphorylation by the knockout and overexpression of p110y. It is possible that $\mathrm{p} 110 \mathrm{y}$ is necessary for $\mathrm{p} 38$ MAPK activation, while overexpressing p110y results in saturation or compensation of the pathway leading to similar impairment of p38 MAPK activation. Overexpression of p110y additionally impaired depotentiation which also involves p38 MAPK signaling [13]. However, since a similar impairment of p38 MAPK activation in Pik3 $\mathrm{cg}^{-/-}$mice was not associated with disruption of depotentiation, the impaired depotentiation in p110y overexpressing slices is likely due to disruption of an additional signaling pathway specifically involved in depotentiation. Further studies are required to establish the underlying mechanisms.

A significant amount of evidence has indicated that hippocampal LTD contributes to learning and memory [14-19]. Various genetic and pharmacological studies have shown that animals having impaired LTD show various behavioral phenotypes, some showing similar impairment in water maze task as the present study $[16,18]$. There seems to be differential role of each pathways required for LTD in various behaviors, and these behavioral phenotypes cannot be simply classified as a general result of LTD impairment. Both p110y knockout and overexpression resulted in LTD impairment while these two opposite manipulations resulted in different behavioral phenotypes. Spatial learning during initial training in $\mathrm{Pik} 3 \mathrm{Cg}^{-1-}$ mice was intact but reversal learning was impaired [9]. In contrast, p110 $\gamma$-overexpressed mice showed impairment in spatial learning during initial training. These results indicate that $\mathrm{p} 110 \mathrm{y}$ is multi-functional, required for spatial learning and reversal of the initial spatial memory in different ways. The role of hippocampal depotentiation in learning and memory is less clearly established. Therefore, we cannot exclude the possibility that impaired depotentiation might be involved in the spatial learning deficit in the p110y-overexpressing mice.

In conclusion, our present findings have added further evidence to support a role for PI3K $\gamma$ in both NMDARLTD and hippocampus-dependent learning and memory.

\section{Competing interests}

The authors declare that they have no competing interests.

\section{Authors' contributions}

$J H C$ and GCB carried molecular studies and drafted the manuscripts. PP performed electrophysiology experiments. SES and SJK performed molecular studies. YL and SHA performed behavioral experiments. CSL, YSL and GLC revised the manuscript critically for important intellectual contents. BKK conceptualized the hypothesis, supervised research and finalized the manuscript. All authors read and approved the final manuscript.

\section{Acknowledgments}

This work was supported by the National Research Foundation of Korea (NRF) grant funded by the Korea government (MSIP) (National Honor Scientist Program: 2012R1A3A1050385).

\section{Author details}

${ }^{1}$ Department of Biological Sciences, College of Natural Sciences, Seoul National University, Bldg 504 Rm 202, 599 Gwanangno, Gwanak-gu, Seoul 151-747, Korea. ${ }^{2}$ Department of Brain and Cognitive Sciences, College of Natural Sciences, Seoul National University, Seoul, Korea. ${ }^{3}$ Department of Life Science, Chung-Ang University, Seoul, Korea. ${ }^{4}$ Centre for Synaptic Plasticity, School of Physiology and Pharmacology, University of Bristol, Bristol BS8 1TD, UK.

Received: 15 September 2014 Accepted: 23 October 2014

Published online: 06 November 2014

\section{References}

1. Fry MJ: Structure, regulation and function of phosphoinositide 3-kinases. Biochim Biophys Acta 1994, 1226:237-268.

2. Rückle T, Schwarz MK, Rommel C: PI3Kgamma inhibition: towards an "aspirin of the 21st century"? Nat Rev Drug Discov 2006, 5:903-918.

3. Whitman M, Kaplan DR, Schaffhausen B, Cantley L, Roberts TM: Association of phosphatidylinositol kinase activity with polyoma middle-T competent for transformation. Nature 1985, 315:239-242.

4. Opazo P, Watabe AM, Grant SGN, O'Dell TJ: Phosphatidylinositol 3-kinase regulates the induction of long-term potentiation through extracellular signal-related kinase-independent mechanisms. J Neurosci Off J SoC Neurosci 2003, 23:3679-3688.

5. Lin CH, Yeh SH, Lin CH, Lu KT, Leu TH, Chang WC, Gean PW: A role for the $\mathrm{PI}-3$ kinase signaling pathway in fear conditioning and synaptic plasticity in the amygdala. Neuron 2001, 31:841-851.

6. Hou L, Klann E: Activation of the phosphoinositide 3-kinase-Akt-mammalian target of rapamycin signaling pathway is required for metabotropic glutamate receptor-dependent long-term depression. J Neurosci Off J SoC Neurosci 2004, 24:6352-6361.

7. Daw MI, Bortolotto ZA, Saulle E, Zaman S, Collingridge GL, Isaac JTR: Phosphatidylinositol 3 kinase regulates synapse specificity of hippocampal long-term depression. Nat Neurosci 2002, 5:835-836.

8. Chen X, Garelick MG, Wang H, Lil V, Athos J, Storm DR: PI3 kinase signaling is required for retrieval and extinction of contextual memory. Nat Neurosci 2005, 8:925-931.

9. Kim J-I, Lee H-R, Sim S, Baek J, Yu N-K, Choi J-H, Ko H-G, Lee Y-S, Park S-W, Kwak C, Ahn S-J, Choi SY, Kim H, Kim K-H, Backx PH, Bradley CA, Kim E, Jang D-J, Lee K, Kim SJ, Zhuo M, Collingridge GL, Kaang B-K: PI3KY is required for NMDA receptor-dependent long-term depression and behavioral flexibility. Nat Neurosci 2011, 14:1447-1454.

10. Zhu JJ, Qin Y, Zhao M, Van Aelst L, Malinow R: Ras and Rap control AMPA receptor trafficking during synaptic plasticity. Cell 2002, 110:443-455.

11. Zhu Y, Pak D, Qin Y, McCormack SG, Kim MJ, Baumgart JP, Velamoor V, Auberson YP, Osten P, van Aelst L, Sheng M, Zhu JJ: Rap2-JNK removes synaptic AMPA receptors during depotentiation. Neuron 2005, 46:905-916.

12. Bos J , de Rooij J, Reedquist KA: Rap1 signalling: adhering to new models. Nat Rev Mol Cell Biol 2001, 2:369-377.

13. Liang $Y-C$, Huang $C-C$, Hsu K-S: A role of p38 mitogen-activated protein kinase in adenosine $A_{1}$ receptor-mediated synaptic depotentiation in area CA1 of the rat hippocampus. Mol Brain 2008, 1:13.

14. Kemp A, Manahan-Vaughan D: Hippocampal long-term depression: master or minion in declarative memory processes? Trends Neurosci 2007, 30:111-118.

15. Manahan-Vaughan D, Braunewell KH: Novelty acquisition is associated with induction of hippocampal long-term depression. Proc Natl Acad Sci U S A 1999, 96:8739-8744.

16. Ge Y, Dong Z, Bagot RC, Howland JG, Phillips AG, Wong TP, Wang YT: Hippocampal long-term depression is required for the consolidation of spatial memory. Proc Natl Acad Sci U S A 2010, 107:16697-16702. 
17. Etkin A, Alarcón JM, Weisberg SP, Touzani K, Huang YY, Nordheim A, Kandel ER: A role in learning for SRF: deletion in the adult forebrain disrupts LTD and the formation of an immediate memory of a novel context. Neuron 2006, 50:127-143.

18. Brigman JL, Wright T, Talani G, Prasad-Mulcare S, Jinde S, Seabold GK, Mathur P, Davis Ml, Bock R, Gustin RM, Colbran RJ, Alvarez VA, Nakazawa K, Delpire E, Lovinger DM, Holmes A: Loss of GluN2B-containing NMDA receptors in CA1 hippocampus and cortex impairs long-term depression, reduces dendritic spine density, and disrupts learning. J Neurosci Off J Soc Neurosci 2010, 30:4590-4600.

19. Nicholls RE, Alarcon JM, Malleret G, Carroll RC, Grody M, Vronskaya S, Kandel ER: Transgenic mice lacking NMDAR-dependent LTD exhibit deficits in behavioral flexibility. Neuron 2008, 58:104-117.

doi:10.1186/s13041-014-0078-6

Cite this article as: Choi et al:: Effects of PI3KY overexpression in the hippocampus on synaptic plasticity and spatial learning. Molecular Brain 2014 7:78.

\section{Submit your next manuscript to BioMed Central and take full advantage of:}

- Convenient online submission

- Thorough peer review

- No space constraints or color figure charges

- Immediate publication on acceptance

- Inclusion in PubMed, CAS, Scopus and Google Scholar

- Research which is freely available for redistribution 